\title{
Water structure at the interface of alcohol monolayers as predicted by computational vibrational sum-frequency generation spectroscopy
}

Daniel R. Moberg, 1,a) Qin Li, ${ }^{1, b)}$ Sandeep K. Reddy, 1,b) and Francesco Paesani ${ }^{1,2,3, c)}$

1) Department of Chemistry and Biochemistry, University of California San Diego, La Jolla, California 92093, United States

2) Materials Science and Engineering, University of California San Diego, La Jolla, California 92093, United States

3) San Diego Supercomputer Center, University of California San Diego, La Jolla, California 92093, United States

In this study, we investigate the structure of water at the interface of three longchain alcohol monolayers differing in alkyl chain length through molecular dynamics simulations combined with modeling of vibrational sum-frequency generation (vSFG) spectra. The effects of alkyl chain parity on interfacial water is examined through extensive analysis of structural properties, hydrogen bonding motifs, and spectral features. Besides providing molecular-level insights into the structure of interfacial water, this study also demonstrates that, by enabling direct comparisons with experimental vSFG spectra, computational spectroscopy may be used to test and validate force fields commonly used in biomolecular simulations. The results presented here can thus serve as benchmarks for both further investigations to characterize ice nucleation induced by alcohol monolayers and refinement of popular biomolecular force fields.

\footnotetext{
a) Electronic mail: drmoberg@ucsd.edu Contributed equally to this work

b) Contributed equally to this work

c) Electronic mail: fpaesani@ucsd.edu
} 


\section{INTRODUCTION}

Although sea spray aerosol (SSA) represents a small fraction of the planet's aerosol concentration, it is shown to play an important role in regulating our climate system. 1 Specifically, SSA particles can have significant impacts on various atmospheric processes by scattering and/or absorbing solar radiation, promoting cloud formation, and catalyzing or suppressing chemical reactions. ${ }^{2}[\underline{6}$ A precise understanding of how SSA mediates atmospheric processes requires quantitative information on both physical and chemical properties of the SSA particles. SSA particles have been found to contain a diverse mixture of components, including inorganic salts, small organic molecules, and biological material (e.g., viruses, bacteria, and vesicles) ${ }^{7}$ Saccharides, carboxylic acids, alcohols, and alkanes have all been identified in SSA particles using various experimental techniques such as mass spectrometry and vibrational spectroscopy $\left[\frac{611}{15}\right.$

Previous studies demonstrated that surfactant molecules with hydroxyl-containing head-

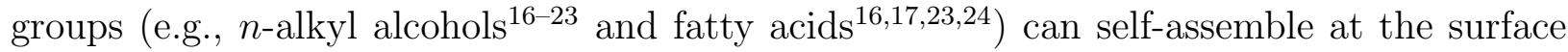
of aqueous solutions into two-dimensional monolayers. As their lattice parameters nearly match those of the basal plane of ice $\mathrm{I}_{h}$, they are capable of promoting ice formation at temperatures higher than that required for homogeneous nucleation $\left(-36^{\circ} \mathrm{C}\right)$. In particular, it has been observed that long-chain alcohol monolayers are quite efficient in promoting heterogeneous ice nucleation at temperatures below $0{ }^{\circ} \mathrm{C}$. Freezing temperatures measured for various alcohol monolayers generally increase with the length of the alkyl chain ${ }^{20 \mid 23}$ but, interestingly, display a noticeable dependence on whether the number of carbon atoms in the chain is even or odd. Specifically, a freezing temperature close to $0^{\circ} \mathrm{C}$ has been measured for alcohol monolayers with alkyl chains containing 31 carbon atoms $\left(\mathrm{C}_{31} \mathrm{H}_{63} \mathrm{OH}\right)$, while freezing temperatures measured for even-number-chain $\left(\mathrm{C}_{22} \mathrm{H}_{45} \mathrm{OH}-\mathrm{C}_{30} \mathrm{H}_{61} \mathrm{OH}\right)$ alcohol monolayers are around $-8{ }^{\circ} \mathrm{C} ! \frac{16|19| 20}{1 t}$ has been proposed that the interactions between alkyl chains within a monolayer with even and odd numbers of carbon atoms are different, altering both the alignment and orientations of the alcohol headgroups, causing changes in the thermodynamic and ferroelectric properties of interfacial water, and, consequently, affecting freezing temperatures. ${ }^{25}$ The 31-carbon chain in particular has been shown to possess greater efficiency in nucleating ice potentially due to the orientation of the $\mathrm{OH}$ bond as well as a more mobile monolayer allowing for rearrangement to better align with the emerging 
ice lattice. $26 \mid 27$

A powerful spectroscopic technique for probing the structure of alcohol monolayers is vibrational sum-frequency generation spectroscopy (vSFG). As vSFG is sensitive to changes in the second order nonlinear susceptibility, which is nonzero only in noncentrosymmetric systems such as interfaces, it can report on the structure of the alcohol monolayer at the water interface. Since the first experimental vSFG signals were reported, ${ }^{1828}$ vSFG spectra have been measured for various alcohol monolayer interfaces, particularly in the $\mathrm{CH}$ stretching region, $\sqrt[28]{33}$ which provides useful information on the orientation and structure of the alkyl chains. Conversely, the $\mathrm{OH}$ stretching region is better suited for analyzing both the orientation of the hydroxyl headgroups as well as the structure of water at the interface. $\frac{18|22 / 30| 34}{36}$ Due to the sensitivity of the vSFG spectrum to the hydrogen bonding strength of water, the $\mathrm{OH}$ stretching region is also a useful probe for detecting the onset of ice nucleation at alcohol monolayer surfaces. Obvious difficulties, however, arise from the complexity inherent in interpreting the signal from the hydrogen bonding network of water as the signal obtained is considerably less intense and broader due to inhomogeneous broadening than that from the $\mathrm{CH}$ stretching region.22

Often not included in the analysis of vSFG spectra of alcohol monolayers, however, is the possible influence of third order effects arising from the development of net charges at the interface. This charge in turn generates its own electric field that can extend into the bulk phase. It has been shown that interfacial electric fields have a non-negligible influence on the observed SFG signal, adding a third order component to the polarization. Disentangling the second and third order contributions to the vSFG response at charged interfaces is particularly challenging and has recently been the focus of several experimental and theoretical studies on model systems. $\underline{34} \underline{53}$

To provide insights into possible molecular mechanisms responsible for the observed differences in ice nucleation efficiency displayed by different alcohol monolayers, in this study we characterize the structure of water at the interface of alcohol monolayers with alkyl chains of different length, namely, $\mathrm{C}_{16} \mathrm{H}_{33} \mathrm{OH}, \mathrm{C}_{30} \mathrm{H}_{61} \mathrm{OH}$, and $\mathrm{C}_{31} \mathrm{H}_{63} \mathrm{OH}$, hereafter denoted as C16, C30, and C31, respectively. In particular, building upon our recent studies of the air/water ${ }^{54}$ and palmitic acid monolayer/water interfaces, ${ }^{36}$ we combine molecular dynamics (MD) simulations with computational modeling of vSFG spectra to provide a systematic, molecular-level analysis of the interfacial region and identify characteristic hydrogen bonding 
topologies induced by different alcohol monolayers.

\section{A. Computational methods}

Three types of alcohol monolayers were considered in this study, C16, C30, and C31, to investigate differences in chain length and odd vs. even numbered chains. Initial configurations for the MD simulations were obtained from PACKMOL 55 The interactions between alcohol molecules were represented using the OPLS all-atom force field, $\underline{56157}$ while the TIP4P/2005 model was used for liquid water ${ }^{58}$ Lorentz-Berthelot mixing rules were employed to estimate the cross-interactions between water and alcohols. The systems were modeled with two monolayers, with 36 alcohol molecules each, placed at the opposite side of a slab (along the $z$-direction) with 972 water molecules in a fully periodic box with dimensions $\mathrm{L}_{x} \times \mathrm{L}_{y} \times$ $\mathrm{L}_{z}=26.2 \AA \times 26.2 \AA \times 150.0 \AA$. This corresponds to a surface area of $19.07 \AA^{2}$ per molecule that closely resembles the value used in earlier studies. ${ }^{23}$ Our previous study has shown that this slab depth is sufficient to reproduce the experimental vSFG spectra and allowing for a reliable estimate of the underlying local structure of interfacial water $\stackrel{36}{n}$ Two vacuum regions were placed above both monolayers to mimic the air interface. All structural properties were averaged over both interfaces present in the simulation box and all results are shown with respect to the Gibbs dividing surface.

All MD simulations were performed at the classical level using the LAMMPS software package. 59 Each system was equilibrated for 100 ns in the canonical ensemble with the velocity-Verlet algorithm used to integrate the equations of motion with a timestep of 2 fs. The temperature was maintained constant at $298 \mathrm{~K}$ using a global Nosé-Hoover thermostat with a relaxation time of 1 ps $\underline{60161}$ Within $9 \AA$, the electrostatic interactions were computed in real space using Coulomb's law, while long-range electrostatic contributions were calculated in reciprocal space using the particle-particle particle-mesh (PPPM) solver. After equilibration, trajectories for another 100 ns were generated to calculate the structural properties.

In order to probe vibrational properties, 48 configurations for all systems were extracted from the NVT trajectories. The water model was replaced by a flexible q-TIP4P/F model, previously shown to be successful in reproducing the structural and dynamical properties

of liquid water.62[63 Consequently, the timestep was reduced to 0.5 fs to properly describe 
the high-frequency modes in the system. Each configuration was further equilibrated for another 100 ps in the NVT ensemble before generating the trajectories in the microcanonical ensemble (NVE) for 100 ps which were then used to calculate the vSFG response of water.

The resonant vSFG polarization, $P_{i}$, can be expressed as 51

$$
P_{i}=\left[\chi_{i j k}^{(2)}-\phi(0) \chi_{i j k z}^{(3)}\right] E_{j}^{v i s} E_{k}^{I R}
$$

under the assumption that the Debye length is significantly larger than the SFG coherence length. Here, $\chi_{i j k}^{(2)}$ and $\chi_{i j k z}^{(3)}$ are the resonant second-order and third-order susceptibilities of the material, respectively. For this study, $i=x, j=x$, and $k=z$ ( $z$ is defined perpendicular to the interface), which correspond to the polarization conditions of the sum frequency, visible, and infrared beams, respectively, $E_{j}^{v i s}$ and $E_{k}^{I R}$ are the electric fields of the incident visible and infrared lasers, respectively, and $\phi(0)=\phi(z=0)$ is the surface potential at the Gibbs dividing surface defined with the assumption that $\phi(z)=0$ for bulk water $(z=-\infty)$. While $\chi_{i j k}^{(2)}$ is surface specific, the non-surface sensitive $\chi_{i j k z}^{(3)}$ is a bulk property that can further influence the $\chi_{i j k}^{(2)}$ response when $\phi(0)$ deviates from zero. $\frac{36 / 37 / 40 / 41 / 43 \mid 52}{15}$ As shown in Eq. 1, its contribution to the vSFG response arises from the interaction with the electric fields associated with the visible and infrared laser pulses, as well as with the static electric field that is generated by interfacial charges and/or net polarized dipoles. The effective, or observed, signal $\chi_{i j k}^{(e f f)}$ is therefore a mixture of $\chi_{i j k}^{(2)}$ and $\chi_{i j k z}^{(3)}$.

This effective signal is proportional to the square of the nonlinear susceptibility and is a combination of resonant and nonresonant contributions, as well as imaginary and real components,

$$
\left|\chi_{i j k}^{(e f f)}\right|^{2}=\left|\operatorname{Re}\left[\chi_{i j k}^{(2)}\right]+N R 2-\phi(0) \operatorname{Re}\left[\chi_{i j k z}^{(3)}\right]-\phi(0) N R 3\right|^{2}+\left|\operatorname{Im}\left[\chi_{i j k}^{(2)}\right]-\phi(0) \operatorname{Im}\left[\chi_{i j k z}^{(3)}\right]\right|^{2}
$$

In Eq. 2, $N R 2$ and $N R 3$ are the second and third order nonresonant background contributions, both approximated as real, constant values. Following Ref. 64, the imaginary part of the resonant surface sensitive susceptibility, $\chi_{i j k}^{(2)}$, is calculated using the time correlation function formalism,,$\underline{64}$

$$
\begin{aligned}
& \operatorname{Re}\left[\chi_{i j k}^{(2)}(\omega)\right]=\operatorname{Re}\left[\frac{i \omega}{k_{B} T} \int_{0}^{\infty} d t e^{-i \omega t}\left\langle\alpha_{i j}(t) \mu_{k}(0)\right\rangle\right], \\
& \operatorname{Im}\left[\chi_{i j k}^{(2)}(\omega)\right]=\operatorname{Im}\left[\frac{i \omega}{k_{B} T} \int_{0}^{\infty} d t e^{-i \omega t}\left\langle\alpha_{i j}(t) \mu_{k}(0)\right\rangle\right],
\end{aligned}
$$


where $\omega$ is the vibrational frequency, $k_{B}$ is Boltzmann's constant, $\alpha_{i j}$ is the $i j$ component of the system polarizability tensor, and $\mu_{k}$ is the $k$ component of the system dipole moment. $\operatorname{Im}\left[\chi_{x x z}^{(2)}\right]$ can be directly compared to the experimental measurement of $\operatorname{Im}\left[\chi_{S S P}^{(2)}\right]$, where $\mathrm{S}$ and $\mathrm{P}$ denote beam polarizations parallel and perpendicular to the surface, respectively, after correction with the appropriate Fresnel factors. In the present study, however, the reported vSFG spectra do not include Fresnel factor corrections. As shown in earlier studies on the air/water interface, the Fresnel factors are negligible due to being near frequency independent $\underline{65}$

Both the molecular dipole moment and polarizability of water were calculated using the MB- $\mu$ dipole moment and MB- $\alpha$ polarizability tensor surfaces ${ }^{\sqrt{6167}}$ without including explicit short-range two-body corrections, which were developed along with the MB-pol many-body potential energy function. ${ }^{66 / 68]}$ Our earlier studies have shown that MB- $\mu$ and MB- $\alpha$ provide an accurate representation of the electrostatic properties of water and, when combined with an accurate polarizable water model, enable an accurate modeling of the vibrational spectra from small water cluster ${ }^{71}$ to liquid water ${ }^{[67}$, the air/water interface, ${ }^{[54172]}$ and ice. ${ }^{[3}$ As given in Eq. 4 , the calculation of $\operatorname{Im}\left[\chi_{S S P}^{(2)}\right]$ involves self-correlation terms as well as cross-correlation terms. The latter terms were handled with the truncated crosscorrelation formalism ${ }^{\sqrt{74}}$, where the response in Eqs. 3 and 4 are approximated as

$$
\left\langle\alpha_{i j}(t) \mu_{k}(0)\right\rangle=\left\langle\sum_{m} \alpha_{m, i j}(t) \mu_{m, r}(0) g_{\mathrm{sc}}^{3}\left(z_{m}\right)\right\rangle+\left\langle\sum_{m} \sum_{n \neq m} \alpha_{n, i j}(t) \mu_{m, k}(0) g_{\mathrm{sc}}^{3}\left(z_{m}\right) g_{t}\left(r_{m n}, r_{t}\right)\right\rangle
$$

In Eq. 5. $g_{t}\left(r_{m n}, r_{t}\right)$ is a stepwise function that includes cross-correlation between molecules $m$ and $n$ only if they are within a predefined cutoff distance $r_{t}=4.0 \AA$, and $g_{\mathrm{sc}}(z)$ is the screening function

$$
g_{\mathrm{sc}}(z)=\operatorname{sign}(z) \times\left\{\begin{array}{l}
0 \text { if }|z| \leq z_{\mathrm{cl}} \\
\cos ^{2}\left(\frac{\pi\left(|z|-z_{\mathrm{c} 2}\right)}{2\left(z_{\mathrm{c} 1}+z_{\mathrm{c} 2}\right)}\right) \\
1 \text { if } z_{\mathrm{c} 2}<|z|
\end{array} \text { if } z_{\mathrm{c} 1}<|z| \leq z_{\mathrm{c} 2}\right.
$$

designed to smoothly transition from including molecules with full contribution to $\chi_{i j k}^{(2)}$ (above $z_{\mathrm{c} 2}$ from the center of mass of the slab) to eliminating contributions from the bulk (below $z_{\mathrm{c} 1}$ from the center of mass of the slab). This approximation was shown to provide an 
accurate approximation to the vSFG response of the air/water interface and water/palmitic

acid monolayers interface. ${ }^{36 / 54 / 72}$ Following the same procedure used in our earlier study, for the vSFG calculations the alkyl chains of the alcohol molecules were truncated at the third carbon atom to reduce the computational cost associated with the evaluation of dipole moment and polarizability. The calculated $\chi_{S S P}^{(2)}$ was redshifted by $152 \mathrm{~cm}^{-1}$ to account for the frequency shift between the classical vSFG spectrum of liquid water predicted by $\mathrm{q}-\mathrm{TIP} 4 \mathrm{P} / \mathrm{F}$ in the $\mathrm{OH}$ stretching region and the corresponding experimental spectrum, a consequence of neglecting nuclear quantum effects.

\section{RESULTS AND DISCUSSION}

\section{A. Structural properties}

The number density profiles of the water and alcohol molecules are shown in Figure 1 for the C16, C30 and C31 systems. Here, the density profiles of water (thick lines, denoted as $\mathrm{O}_{\mathrm{w}}$ ) and the alcohol group (dotted lines, denoted as $\mathrm{Oh}$ ) are shifted along the $z$-direction to set their Gibbs dividing surfaces as the origin. The Gibbs dividing surface is defined here as the position where the water density has dropped to half of its mean bulk value. As shown in Figure 1, a well-defined boundary is established between the water and alcohol monolayers for the even parity C16 and C30 systems. Similar density profiles of water and alcohol groups are found for both $\mathrm{C} 16$ and C30, with only slight differences just below the Gibbs dividing surface, indicating that alkyl chain length has little effect on the water density in even-numbered alcohol monolayers.

In contrast, found in the density profile of C31 are density fluctuations extending over the water density deeper into the bulk and farther into the alcohol monolayer as compared to the C16 and C30 systems. Additionally, there exists a step-wise progression in the density while moving from the bulk through the Gibbs dividing surface and into the alcohol monolayer. This broader distribution is possibly due to an overall less rigid monolayer.26|27 The C31 alcohol headgroup also shows a more disrupted distribution compared to the C16 and C30 monolayers, with the distribution extending farther into the bulk water phase. When combined with the stepwise water distribution, the picture that emerges is that of the C31 monolayer having more out-of-plane motion and extending into the water bulk phase, 


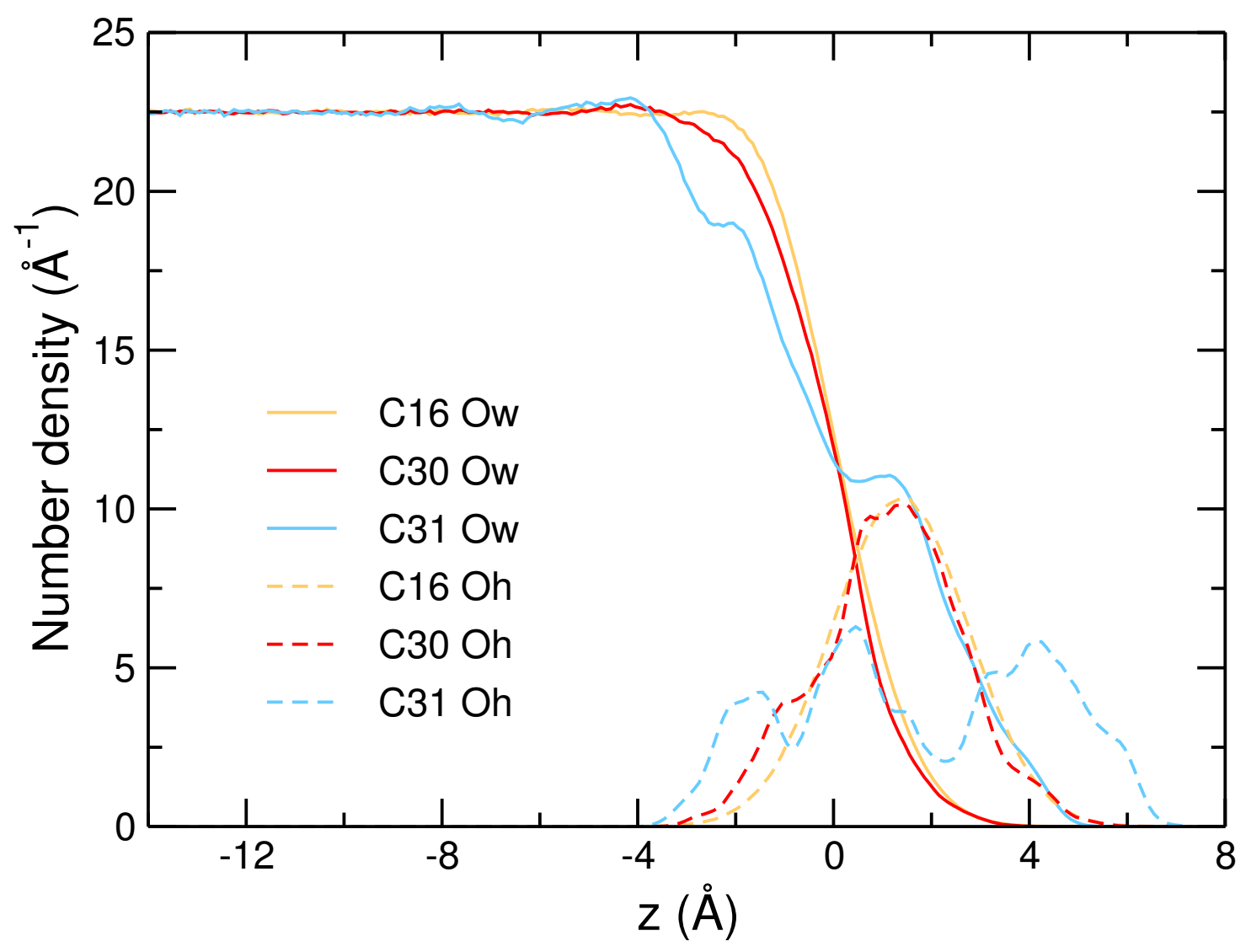

FIG. 1: Number density profiles of oxygens of water and alcohol group along the surface normal for three long-chain alcohol monolayers.

whereas the $\mathrm{C} 16$ and $\mathrm{C} 30$ monolayers remain above the water surface with a more ordered structure.

The methyl groups may display enhanced out-of-plane motion due to the rotation around the neighboring $\mathrm{C}-\mathrm{C}$ bond or the formation of gauche conformations at the terminal end of the chain. In order to quantify the degree of motion experienced in each monolayer, the order parameter $S_{i}$ was calculated for each system, as defined by

$$
S_{i}=\frac{3\left\langle\cos ^{2} \theta_{i}\right\rangle-1}{2}
$$

where $\theta_{i}$ is the angle between the surface normal (the $z$ direction) and the vector connecting neighboring carbon atoms $\left(\mathrm{C}_{i-1}\right.$ and $\left.\mathrm{C}_{i+1}\right)$, with the average taken over all alcohol molecules in the trajectory. As shown in Figure $2 \mathrm{a}, S_{i}$ is nearly constant in the C16 and C30 systems, excluding the terminal carbon atoms, indicating that the monolayers possess a high degree of order and minimal out-of-plane motion. By contrast, the alkyl chains of C31 exhibit some disorder due to the relatively higher sliding of the alcohol molecules into the bulk water. In 

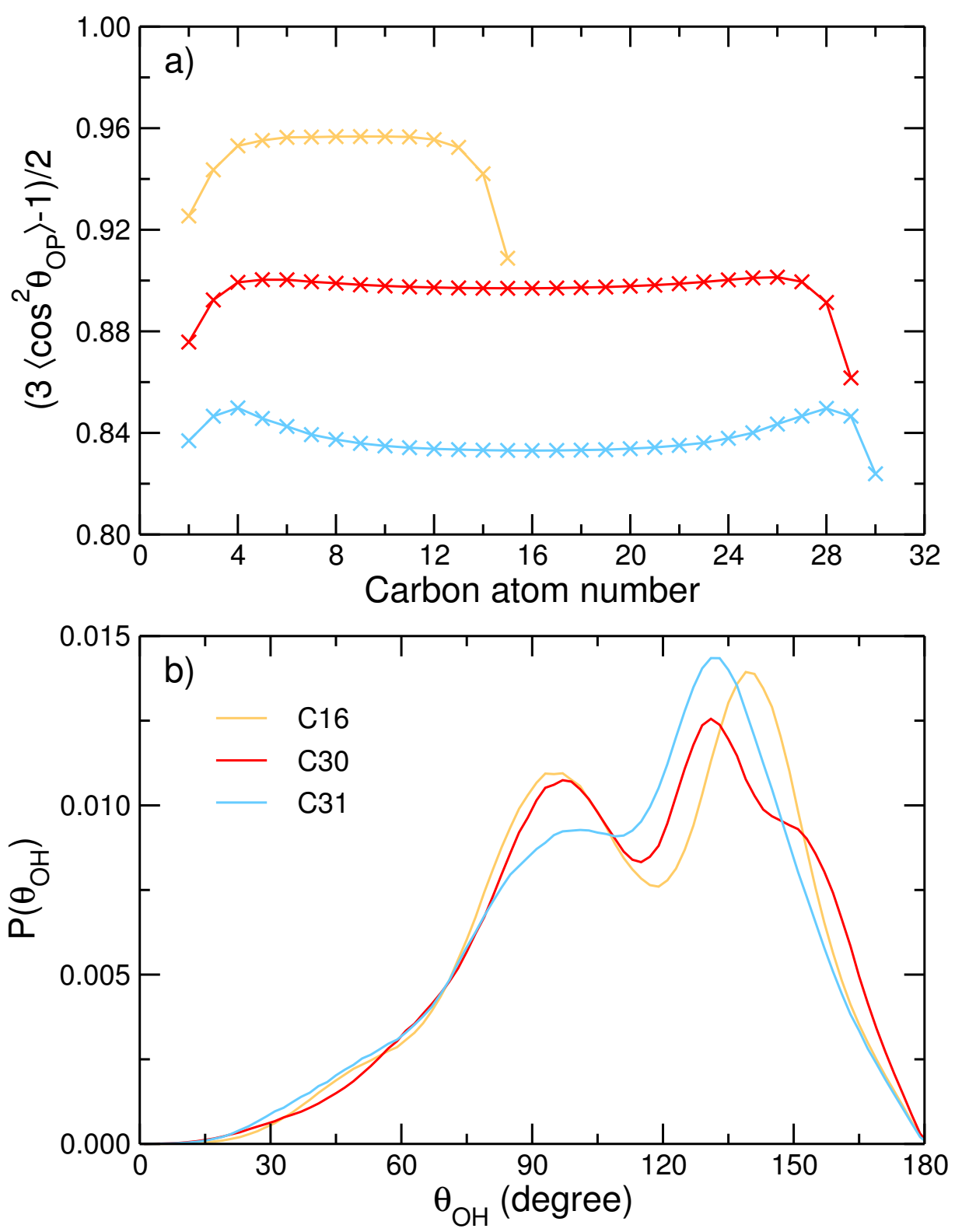

FIG. 2: a) Local order parameter b) probability distribition of alcohol group orientation with respect to the surface normal calculated for three long-chain alcohol monolayers.

all systems, the alcohol molecules are not completely upright and the average value of $S_{i}$ over all carbon atoms is $0.95,0.90$ and 0.84 (roughly equal to angles of 10.6, 15.3 and $19.2^{\circ}$ with respect to the surface normal) for the C16, C30 and C31 systems respectively. In other words, while the change in average angle from C16 to C30 is due to increased flexibility of the alkyl chain as a result of the doubling in length, the change in average angle from C30 to C31 is nearly the same despite being only a single methyl group longer.

Figure $2 \mathrm{~b}$ shows the distribution of the orientation of alcohol $\mathrm{OH}$ groups with respect 
to the surface normal for the three monolayer systems. Each distribution is characterized by two peaks separated by roughly $40^{\circ}$, with the majority of the angles appearing at above $80^{\circ}$, indicating that the alcohol $\mathrm{OH}$ groups point down towards the bulk water. In addition, the distribution corresponding to C30 displays a shoulder at higher angles. The maximum of the first peak appears at $\sim 95^{\circ}$ which suggests that the alcohol $\mathrm{OH}$ groups are parallel to the water surface while that of the second peak is in the range of $130-140^{\circ}$ for all systems. Among the three systems, the amplitude of the first band is smallest for C31, with the second peak compensated with a higher intensity. The distinct behavior of C31 is due to the flexible motion of the monolayers along the surface normal. ${ }^{2012326}$

In order to characterize the local hydration structure around the alcohol headgroups, pair correlation functions, $g(r)$, reporting on the probability of finding two atoms A and $\mathrm{B}$ at a given distance $r_{A-B}$ are also analyzed. Figure 3 shows $\mathrm{g}(\mathrm{r})$ between the hydroxyl hydrogen of an alcohol headgroup and the oxygen atom of a water molecule, referred to as $\mathrm{g}\left(\mathrm{O}_{\mathrm{W}}-\mathrm{H}_{\mathrm{O}}\right)$ in panel a, and that between the hydroxyl oxygen of the alcohol headgroup and the hydrogen of a water molecule, denoted as $\mathrm{g}\left(\mathrm{H}_{\mathrm{W}}-\mathrm{O}_{\mathrm{H}}\right)$ in panel b. Note that, by reporting on the distributions of water molecules around the alcohol headgroups, both $\mathrm{g}(\mathrm{r})$ provide information on the strength of water-alcohol hydrogen bonds. The positions of the first and second peaks in Figure $3 \mathrm{a}$ and $3 \mathrm{~b}$ are effectively independent of the alkyl chain length. The fact that the shape and position of the second maximum in both $\mathrm{g}\left(\mathrm{O}_{\mathrm{W}}-\mathrm{H}_{\mathrm{O}}\right)$ and $\mathrm{g}\left(\mathrm{H}_{\mathrm{W}}-\mathrm{O}_{\mathrm{H}}\right)$ remain unchanged suggests that either the water structure beyond the first coordination shell of the alcohol headgroup is unaffected or the structural changes induced by the monolayers are equal in the second coordination shell around alcohol headgroup. However, the latter is unlikely as the hydrogen bonding interactions are not strong enough to induce structural perturbation in the second coordination shell.

Since the shape of the first coordination shell is different between $\mathrm{g}\left(\mathrm{O}_{\mathrm{W}}-\mathrm{H}_{\mathrm{O}}\right)$ and $\mathrm{g}\left(\mathrm{H}_{\mathrm{W}^{-}}\right.$ $\mathrm{O}_{\mathrm{H}}$ ), the number of water molecules around the alcohol headgroups for the three alcohols are also compared. Figure $3 a$ and $b$ show coordination numbers as a function of $r$ for all three systems. At the first minimum in $\mathrm{g}(\mathrm{r})$, the number of water molecules around the hydroxyl oxygen and hydroxyl hydrogen are 1.05 and 0.80 respectively, and their value does not vary with changes in alkyl length. Interestingly, the number of water molecules within the first coordination shell of $\mathrm{g}\left(\mathrm{H}_{\mathrm{W}}-\mathrm{O}_{\mathrm{H}}\right)$ are higher despite the first peak's maximum intensity being lower than $\mathrm{g}\left(\mathrm{O}_{\mathrm{W}}-\mathrm{H}_{\mathrm{O}}\right)$. This can be interpreted in terms of the vSFG spectra, discussed 


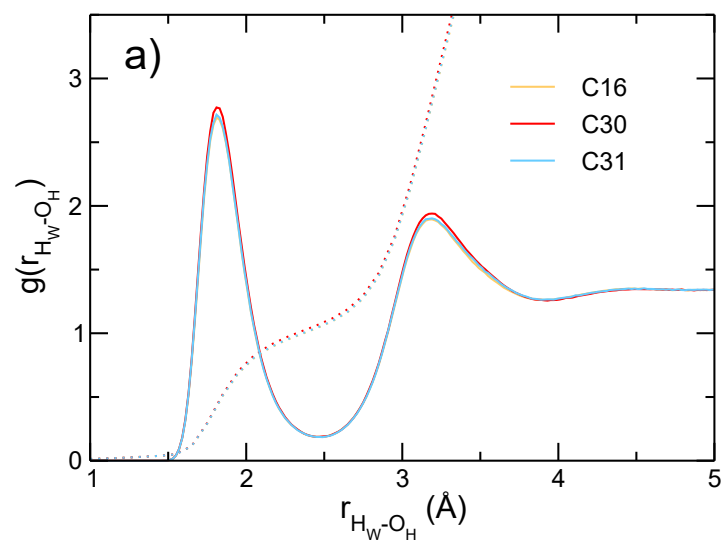

c)

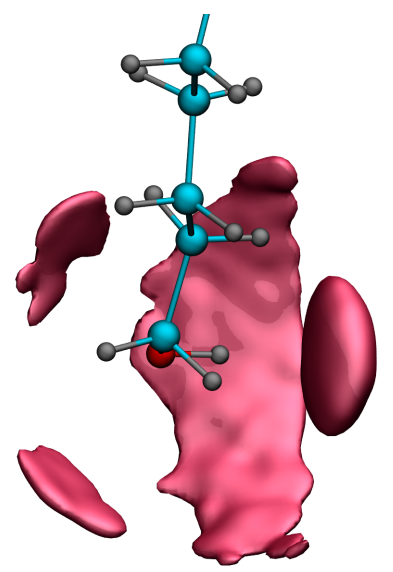

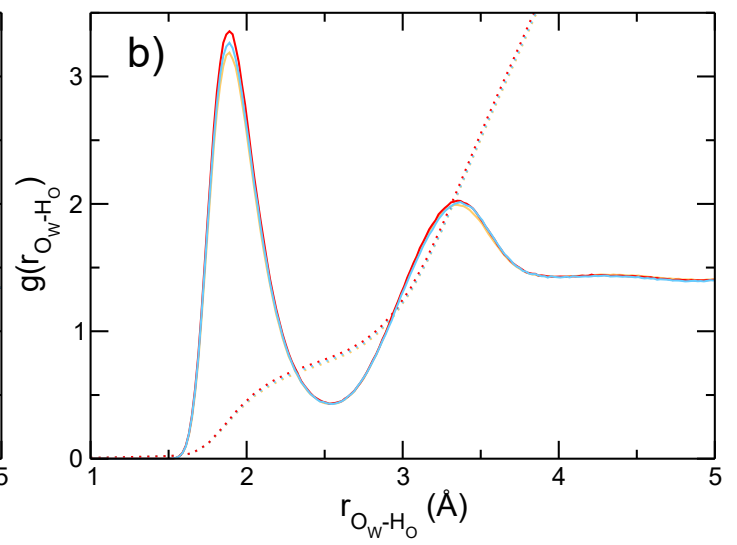

d)

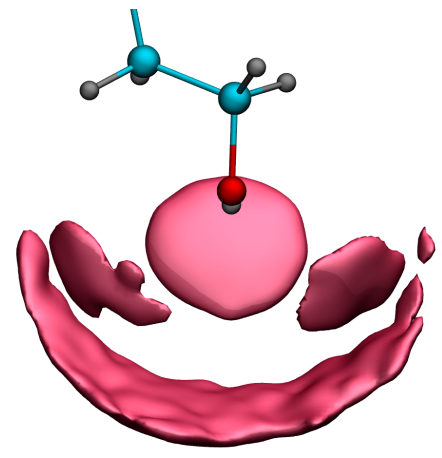

FIG. 3: a) and b) Radial distribution functions between water $\left(\mathrm{O}_{\mathrm{W}}\right.$ ans $\left.\mathrm{H}_{\mathrm{W}}\right)$ and alcohol headgroups $\left(\mathrm{H}_{\mathrm{O}}\right.$ and $\left.\mathrm{O}_{\mathrm{H}}\right)$. Dotted lines display the coordination number. Spatial distribution function showing the water density from c) top and d) side views around the alcohol headgroup.

in the next section. As there are more water molecules donating hydrogen bonds to the alcohol headgroups compared to water molecules accepting a hydrogen bond from alcohol headgroups (Fig. 3k), the donating hydrogen bond waters contribute more to the vSFG response.

\section{B. Vibrational sum frequency generation spectra}

The absorptive, imaginary part of the sum frequency signal, $\operatorname{Im}\left[\chi_{S S P}^{(2)}(\omega)\right]$, which depends on the $z$ component of the system dipole moment, $\mu_{z}$, can be interpreted in terms of the orientation of the transition dipole moment of molecules at the interface. It is important to note that the analysis of the experimental vSFG spectrum is complicated by the 
spectral overlap of the $\mathrm{OH}$ stretching vibrations of water molecules with the $\mathrm{OH}$ stretching vibrations of the alcohol group at the monolayer. ${ }^{22 \mid 35}$ The spectra presented here examine only the signal from the water molecules in order to discern the effect the monolayers have on the water molecules at the interface.

Although the vSFG signal is sensitive to the interfacial region, the depth of this region depends on the properties of the system under investigation, which is difficult to determine precisely. Simulations and experiments tend to agree on a depth of around 4-5 $\AA$ for the

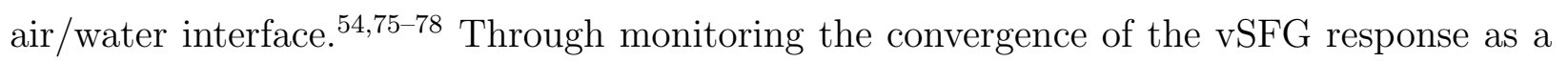
function of the depth of the slab included in the truncated cross correlation calculation, the contribution of water at the interface to the vSFG signal can be determined systematically. By adjusting the values of $z_{c 1}$ and $z_{c 2}$ used in the screening function in Eq. 6, the height of the bulk water phase included in the calculation of $\operatorname{Im}\left[\chi_{S S P}^{(2)}(\omega)\right]$ with Eq. 5 can be fixed to a desired value. Figure 4 shows the $\operatorname{Im}\left[\chi_{S S P}^{(2)}(\omega)\right]$ spectra calculated for the three alcohol monolayers as a function of the depth of the interface region included in the calculations. It is evident that the vSFG spectra converge at approcimately the same distance of $\sim 4.5 \AA$ below the Gibbs dividing surface. Therefore, the alkyl chain length does not influence the convergence of the interfacial region despite the fact that C31 extends deeper than C16 and C30 (Fig. 1). The value of $\sim 4.5 \AA$ also agrees with the results reported for the neat air/water interface $\mathrm{C}^{5477}$ and palmitic acid monolayer on a aqueous subphase. ${ }^{36}$

All $\operatorname{Im}\left[\chi_{S S P}^{(2)}(\omega)\right]$ spectra in Figure 4 show two positive features centered at around 3330

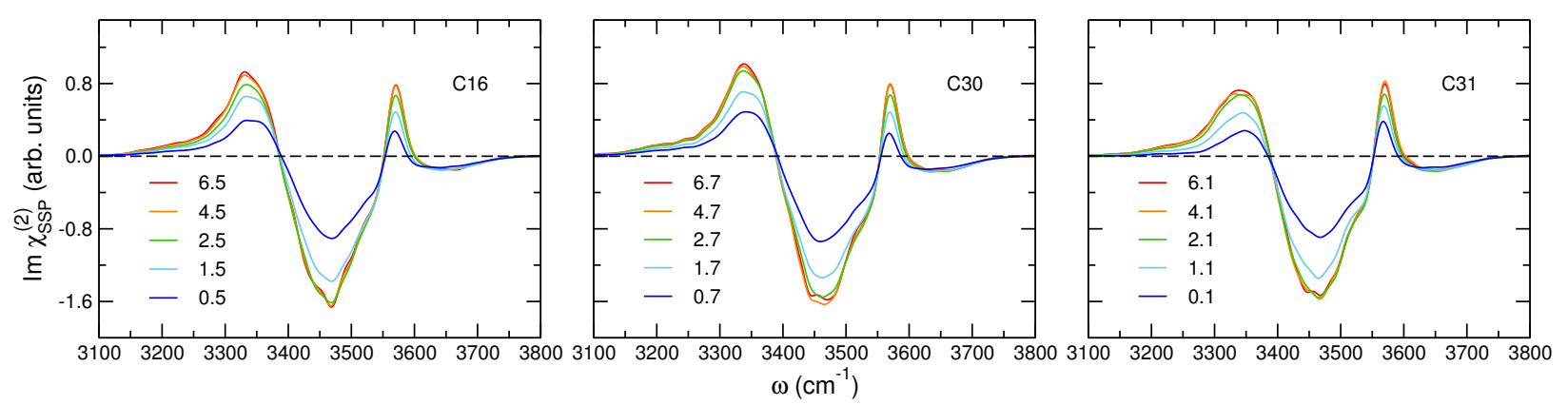

FIG. 4: $\operatorname{Im}\left[\chi_{S S P}^{(2)}\right]$ spectra calculated for water at the interface with alcohol monolayers as a function of the distance from the Gibbs dividing surface included in the spectra calculation. The slab thickness is defined as the distance (in $\AA$ ) from the Gibbs dividing surface along surface normal. 
and $3570 \mathrm{~cm}^{-1}$ and one broad negative band centered at $3470 \mathrm{~cm}^{-1}$. This is in contrast to the imaginary part of the vSFG spectra of the air/water interface ${ }^{7217980}$ and air/fatty acid/water interfaces ${ }^{34 \mid 36}$ which both display only two main features, a sharp positive peak at high frequency and a broad lower frequency band. The sharp high frequency peak, which is $\sim 140 \mathrm{~cm}^{-1}$ redshifted relative to the free $\mathrm{OH}$ peak in the vSFG spectra of the air/water, was also observed in the palmitic acid (PA) monolayer system ${ }^{[36}$ as a result of hydrogen bonding between water and the headgroups of the monolayer. This suggests that water donates hydrogen bonds to the alcohol headgroups, which have similar strengths as those found between water and PA headgroups. However, in VSFG experiments on alcohol monolayers at the air/water interface, this feature is generally not present in the SSP polarization, $\frac{1835}{185}$ though it has been detected in the SPS and PPP cases. ${ }^{22}$ As the peak is shown to be small and the result of cancellations of competing positive and negative signals in Figure 5 , it is likely that the presence of this peak is highly dependent on the force fields used to describe water, alcohol monolayer, and the interactions between them. The presence of the third feature at $3330 \mathrm{~cm}^{-1}$ can be attributed to the disruption of the broad hydrogen-bonded band observed in the vSFG spectra of the neat air/water interface. While in the PA monolayer spectrum this band is redshifted and decreases in intensity, it does not display any positive feature at low frequency. This suggests that the alcohol monolayers perturb the hydrogen-bond network of interfacial water in a fundamentally different way than the PA monolayer.

The vSFG spectra of water at the interface of the three alcohol monolayers shown in Figure 4 are effectively identical. This indicates that the interfacial water structure is distorted from the neat air/water surface by hydrogen bonding with the alcohol groups only, and the lengths of the alkyl groups do not have an appreciable influence on either the water hydrogen bonding structure or the water orientation. Although the local order parameters are marginally different among the three monolayers and the C31 number density shows a broader distribution near the interface, the interactions between the alcohol headgroups and interfacial water molecules are not changed on average. This is supported by the essentially identical radial distributions in Figure 3 that show the solvation shell structures do not change between the different monolayers.

To extract the underlying molecular features that yield different spectral signatures, the full spectra are decomposed into contributions originating from the different hydrogen bonding arrangements between the water and alcohol headgroups. Three different types of water 
molecules are identified in the interfacial region. Two types of water molecules, denoted as $\mathrm{W}_{\mathrm{D}}$ and $\mathrm{W}_{\mathrm{A}}$, correspond to a water molecule either donating or accepting hydrogen bonds to/from the alcohol headgroups, respectively. The third type of water molecules, denoted as WAT, includes water molecules that do not form hydrogen bonds with the alcohol headgroups, though hydrogen bonding may still be and likely is occurring between WAT species. The resulting decomposed vSFG spectra are presented in Figure 5.

The large peak in the vSFG spectrum corresponding to the $\mathrm{W}_{\mathrm{D}}$ water molecules is redshifted by $\sim 50 \mathrm{~cm}^{-1}$ compared to the corresponding peak in the $\mathrm{W}_{\mathrm{A}}$ spectrum, indicating that the water molecules form stronger hydrogen bonds with the alcohol headgroup when donating their hydrogen to $\mathrm{O}_{\mathrm{H}}$ of the headgroup than when accepting a hydrogen from $\mathrm{H}_{\mathrm{O}}$. This agrees with previous CCSD(T)-level coupled cluster calculations of gas phase hydrogen bonded methanol and ethanol complexes suggesting water is a better hydrogen bond donor than alcohols. ${ }^{81}$ This redshift is the source of the three features distinguishable in the full spectrum. The offset of the $\mathrm{W}_{\mathrm{D}}$ and $\mathrm{W}_{\mathrm{A}}$ signals results in two well defined features: the broad positive band at $3330 \mathrm{~cm}^{-1}$ due to the $\mathrm{W}_{\mathrm{D}}$ water molecules and the negative band at $3470 \mathrm{~cm}^{-1}$ containing contributions primarily from the $\mathrm{W}_{\mathrm{A}}$ water molecules. Additional intensity in the negative band is due to the WAT molecules not interacting with the alcohol headgroups. For palmitic acid, the position of the $\mathrm{W}_{\mathrm{D}}$ contributions to the vSFG spectra, witch originate from water molecules donating either the hydroxyl or carbonyl PA headgroups, are strongly blueshifted by $\sim 200 \mathrm{~cm}^{-1}$ from the signal from water molecules donating hydrogen bonds to the alcohol headgroups. ${ }^{36}$ As the two types of palmitic acid $\mathrm{W}_{\mathrm{D}}$

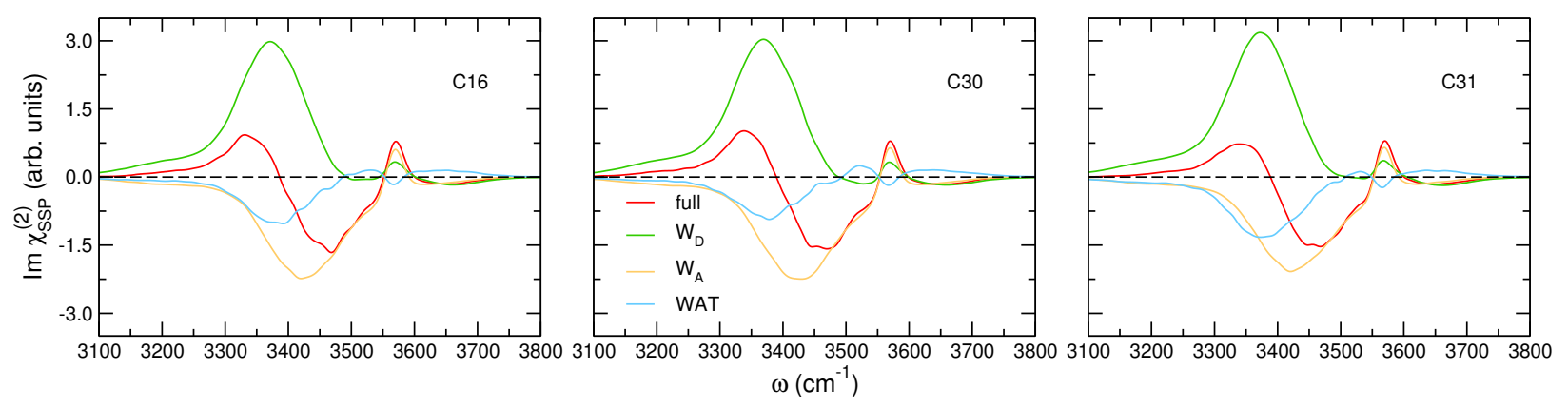

FIG. 5: Decomposition of $\operatorname{Im}\left[\chi_{S S P}^{(2)}(\omega)\right]$ spectra calculated for water at the interface with alcohol monolayer into contributions associated with water molecules donating and accepting hydrogen bonds with alcohol groups. Contribution from the remaining interfacial water molecules is also shown. 
vSFG spectra are both entirely positive and overlap constructively with the sharp positive peak at $3600 \mathrm{~cm}^{-1}$ of $\mathrm{W}_{\mathrm{A}}$, only two features are present in the full PA vSFG spectrum.

The vSFG spectra shown in Figures 4 and 5 included only contributions from the imaginary component of the second order nonlinear susceptibility. However, the experimental alcohol monolayer/water surface may contain an additional third order contribution, a result of the charged interface created by the polar headgroup. The inclusion of third order bulk contributions to the vSFG spectra as well as a nonresonant background are shown in Figure 6. Following Eq. 2, Figure 6a shows the effects of both the nonresonant background and surface potential on the shape of the spectrum. Figure 6 b shows the $\left|\chi_{S S P}^{(e f f)}\right|^{2}$ spectrum with different surface potentials compared against the $\left|\chi_{S S P}^{(2)}\right|^{2}$ spectrum containing no third order terms in the top panel, followed by individual imaginary and real components in the middle and bottom panels. Shown are the spectra for only the C16 system as both the large influence of the $\chi_{S S P P}^{(3)}$ contribution and the similarities between the $\chi_{S S P}^{(2)}$ components for the different alcohol-water systems results in indistinguishable spectra. While the spectra show minimal differences between the different alcohol monolayer systems, the value of the surface potential, $\phi(0)$, has a noticeable effect on the overall lineshape of $\left|\chi_{S S P}^{(e f f)}\right|^{2}$. If the surface potential varies significantly for different alcohols, this would be reflected in differences in measured $\left|\chi_{S S P}^{(e f f)}\right|^{2}$ spectra. The broad change possible in $\left|\chi_{S S P}^{(e f f)}\right|^{2}$ by variation in $\phi(0)$ has been demonstrated recently with surface potential deviations on the order of $10 \mathrm{meV}{ }^{[52]}$ For reference, the surface potential for a palmitic acid monolayer was estimated to be $\sim 0.2 \mathrm{~V} . \underline{36}$ However, as recent experiments on alcohol monolayers find minimal contribution resulting

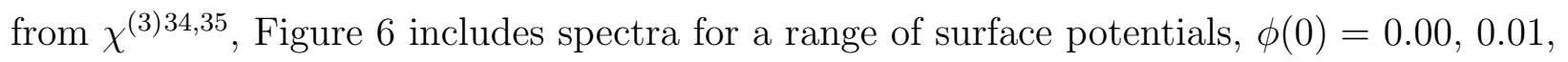
0.03 , and $0.05 \mathrm{~V}$. At larger values above $\sim 0.1 \mathrm{~V}$, the third order contribution dominates the signal, removing any features specific to C16.

Experimental vSFG spectra of decanol, ${ }^{[22}$ hexadeconol,,$\sqrt{35}$ and octadecanol ${ }^{18}$ monolayers on water were previously reported and display generally broad signals in the $\mathrm{OH}$ stretching region, particularly in the low frequency portion of the $\mathrm{OH}$ stretching region, between 3000 and $3550 \mathrm{~cm}^{-1}$. This is in general agreement with the $\left|\chi_{S S P}^{(e f f)}\right|^{2}$ shown in Figure 6 for smaller nonresonant backgrounds and surface potentials. Ref. 35 also measured phase sensitive vSFG spectra of the hexadecanol/water system and found a single negative feature for the imaginary component. They reported the $\left|\chi_{S S P}^{(e f f)}\right|^{2}$ spectrum, showing no positive feature above $\sim 3600 \mathrm{~cm}^{-1}$, at odds with both Refs. 18 and 22. From the analysis reported 

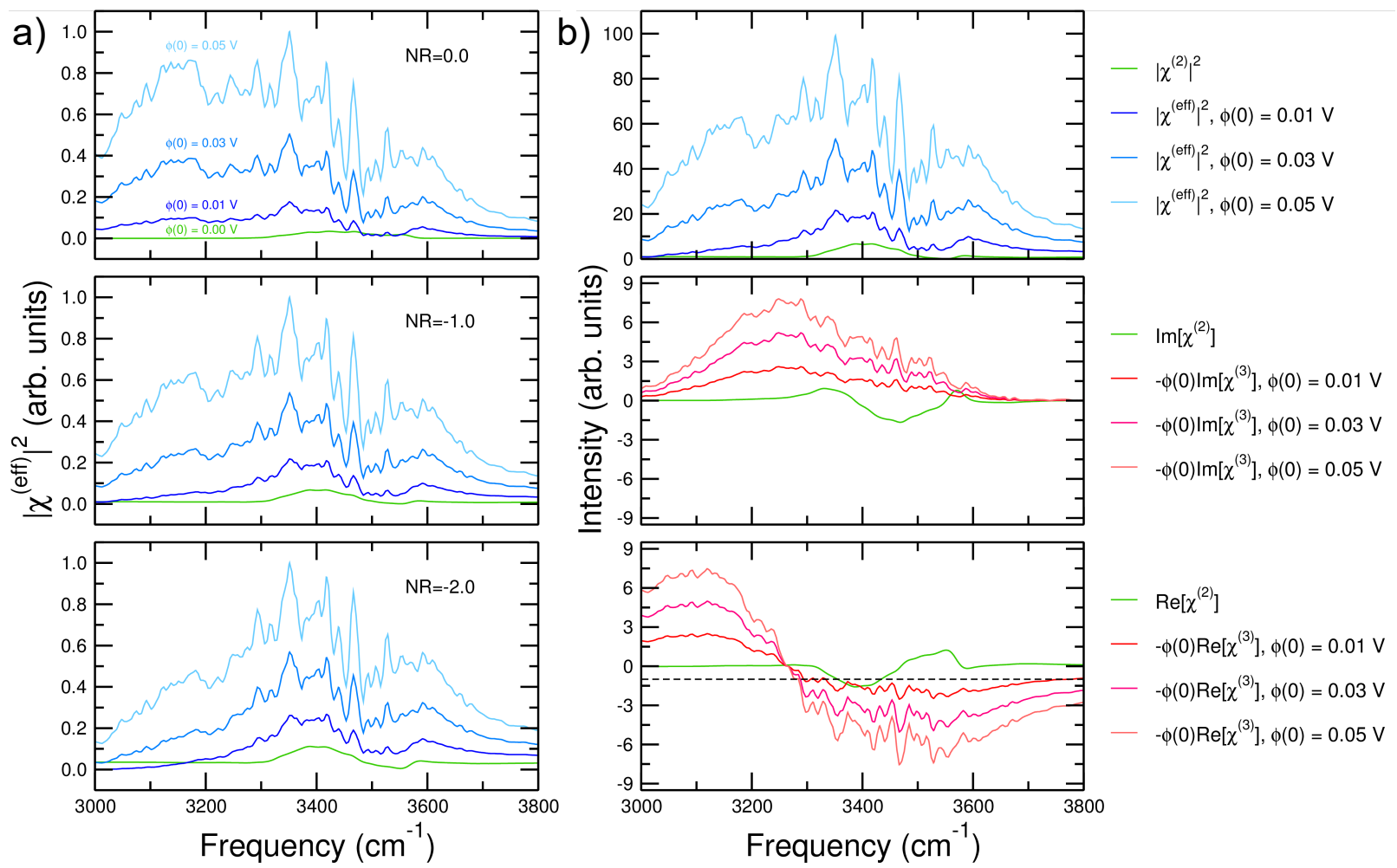

FIG. 6: a) $\left|\chi_{S S P}^{(e f f)}\right|^{2}$ calculated with both second order and third order vSFG contributions at different values of $\phi(0)$ for, from top to bottom, a nonresonant background of $0.0,-1.0$, and -2.0 , respectively, before normalizing to the max intensity of the spectrum for $\phi(0)=$ 0.05 V. b) From top to bottom, $\left|\chi_{S S P}^{(e f f)}\right|^{2}$ compared with $\left|\chi_{S S P}^{(2)}\right|^{2},-\phi(0) \operatorname{Im}\left[\chi_{S S P P}^{(3)}\right]$ compared to $\operatorname{Im}\left[\chi_{S S P}^{(2)}\right]$, and $-\phi(0) \operatorname{Re}\left[\chi_{S S P P}^{(3)}\right]$ compared to $\operatorname{Re}\left[\chi_{S S P}^{(2)}\right]$, with $\mathrm{NR}=-1.0$. The dashed line in the bottom panel indicates the nonresonant background contribution.

in Figure 6, this would be possible only with negligible third order and nonresonant contributions. While the imaginary spectrum in Figure 6b also displays a strong negative component, the experimental spectrum shows no sign of the small positive features at low and high frequency. The source of these discrepancies between experimental and calculate vSFG spectra may be due to the use of empirical force fields (TIP4P/2005 for water and OPLS for the alcohol monolayers). Improvements to the treatment of the alcohol potentials and interactions between the water and alcohol species should be a priority in refining the accuracy of the calculated vSFG spectrua. As the analysis in Figure 5 shows, the competing positive and negative contributions require accurate intensities and peak positions to quantitatively reproduce the experimental vSFG spectra. 


\section{CONCLUSIONS}

Classical MD simulations were performed to model the interface between water and three different long-chain alcohol monolayers. As alcohol monolayers have been shown to promote ice nucleation above the homogeneous nucleation temperature, it is important to determine how different alcohols affect the topmost layer of water. To characterize the structure of water at the interface and the effects induced by the alcohol monolayers, interfacial structural properties and vSFG spectra of water in the $\mathrm{OH}$ stretching region were investigated. The analysis of the density profiles and local order parameters indicates that the alkyl chain length has only a minimal effect on the structure of the interface and monolayer for the C16 and C30 alcohols. Conversely, the odd numbered C31 alcohol displays a relatively wider distribution and large decrease in the stability of the monolayer, in agreement with previous studies on the dependence of length and parity of alkyl chains on the structure of alcohol monolayers. ${ }^{20 \mid 23}$ Comparison of the vSFG spectra of water calculated at the interface with the three different alcohol monolayers, however, demonstrates that the spectral features and spectral shape remain approximately identical to each other. This suggests that the nature of hydrogen bonding between water molecules and the alcohol monolayers, as well as the orientation of the water molecules are induced by the local structural environment around the interfacial water molecules and are not influenced by the long-chain alkyl groups. This is supported by the analysis of the radial distribution functions calculated between the alcohol headgroups and the water molecules, showing no change in peak positions and only slight variation in peak heights. These results support the notion that the C31 monolayer, which has been shown to be the most effective alcohol monolayer in nucleating ice, is able to achieve this solely through having a more flexible and less stable monolayer structure ${ }^{26 \mid 27}$ This is unrelated to interactions with the water surface, as the long-chain alcohols investigated in this work display no differences in their alcohol-water distributions or vSFG spectra.

The third order bulk contribution of the water can dramatically influence the observed vSFG spectra depending on the magnitude of the surface potential. It is still unclear the degree to which the third order and non-resonant background components can obscure the second order signal. While earlier experiments on alcohol monolayers ${ }^{18 / 22}$ appear to display large contributions from sources other than the resonant second order term, recent phase

sensitive experiments $s^{3435}$ that isolated the imaginary component report minimal interference 
from both nonresonant and third order terms. Inclusion of $\chi^{(3)}$ can reproduce some aspects of the spectra measured previously, including the broad feature below $3500 \mathrm{~cm}^{-1}$ and the feature above $\left.3600 \mathrm{~cm}^{-1} ! 1822\right]$ For a more robust comparison, a thorough treatment of the $\chi^{(3)}$ contribution evaluated under the same simulation conditions as $\chi^{(2)}$ with nuclear quantum effects included should be considered. The vSFG spectra presented here also provide a useful benchmark for further investigations in the region around the freezing point to understand the mechanism of ice nucleation induced by the alcohol monolayers, a subject for future studies.

\section{ACKNOWLEDGEMENTS}

This research was supported by the National Science Foundation through grant no. CHE1453204 and used resources of the Extreme Science and Engineering Discovery Environment (XSEDE) ${ }^{826}$ which is supported by the National Science Foundation through grant no. ACI1053575, under allocation TG-CHE110009. 


\section{REFERENCES}

${ }^{1}$ E. Lewis, R. Lewis, R. Sparks, K. Karlstrom, C. Hawkesworth, and S. Schwartz, Sea Salt Aerosol Production: Mechanisms, Methods, Measurements, and Models - A Critical Review, Geophysical Monograph Series (Wiley, 2004).

${ }^{2}$ D. M. Murphy, J. R. Anderson, P. K. Quinn, L. M. McInnes, F. J. Brechtel, A. M. Kreidenweis, S. M. vand Middlebrook, M. Pósfai, D. S. Thomson, and P. R. Buseck, Nature 392, 62 (1998).

${ }^{3}$ M. P. Meyers, P. J. DeMott, and W. R. Cotton, J. Appl. Meteorol. 31, 708 (1992).

${ }^{4}$ T. Koop, B. Luo, A. Tsias, and T. Peter, Nature 406, 611 (2000).

${ }^{5}$ Y. Kumaki, K. Kawano, K. Hikichi, T. Matsumoto, and N. Matsushima, Biochem. Biophys. Res. Commun. 371, 5 (2008).

${ }^{6}$ R. E. Cochran, O. S. Ryder, V. H. Grassian, and K. A. Prather, Acc. Chem. Res. 50, 599 (2017).

${ }^{7}$ D. C. Blanchard and L. D. Syzdek, J. Geophys. Res. 77, 5087 (1972).

${ }^{8}$ A. P. Ault, R. C. Moffet, J. Baltrusaitis, D. B. Collins, M. J. Ruppel, L. A. CuadraRodriguez, D. Zhao, T. L. Guasco, C. J. Ebben, F. M. Geiger, et al., Environ. Sci. Technol. 47, 5603 (2013).

${ }^{9}$ B. Gantt and N. Meskhidze, Atmos. Chem. Phys. 13, 3979 (2013).

${ }^{10}$ P. K. Quinn, D. B. Collins, V. H. Grassian, K. A. Prather, and T. S. Bates, Chem. Rev. 115, 4383 (2015).

${ }^{11}$ L. M. Russell, L. N. Hawkins, A. A. Frossard, P. K. Quinn, and T. S. Bates, Proc. Natl. Acad. Sci. USA 107, 6652 (2009).

${ }^{12}$ L. N. Hawkins and L. M. Russell, Adv. Meteorol. 14, 1 (2010).

${ }^{13}$ P. Q. Fu, K. Kawamura, J. Chen, B. Charrière, and R. Sempéré, Biogeosciences 10, 653 (2013).

${ }^{14}$ P. K. Quinn, T. S. Bates, K. S. Schulz, D. J. Coffman, A. A. Frossard, L. M. Russell, W. C. Keene, and D. J. Kieber, Nat. Geosci. 7, 228 (2014).

${ }^{15}$ R. E. Cochran, O. Laskina, J. V. Trueblood, A. D. Estillore, H. S. Morris, T. Jayarathne, C. M. Sultana, C. Lee, P. Lin, J. Laskin, et al., Chem 2, 655 (2017).

${ }^{16}$ M. Gavish, R. Popovitz-Biro, M. Lahav, and L. Leiserowitz, Science 250, 973 (1990). 
${ }^{17}$ D. Jacquemain, F. Leveiller, S. P. Weinbach, M. Lahav, L. Leiserowitz, K. Kjaer, and J. Als-Nielsen, J. Am. Chem. Soc. 113, 7684 (1991).

${ }^{18}$ Q. Du, R. Superfine, E. Freysz, and Y. R. Shen, Phys. Rev. Lett. 70, 2313 (1993).

${ }^{19}$ J. Majewski, R. Popovitz-Biro, K. Kjaer, J. Als-Nielsen, M. Lahav, and L. Leiserowitz, J. Phys. Chem. 98, 4087 (1994).

${ }^{20}$ R. Popovitz-Biro, J. L. Wang, J. Majewski, E. Shavit, L. Leiserowitz, and M. Lahav, J. Am. Chem. Soc. 116, 1179 (1994).

${ }^{21}$ L. H. Seeley and G. T. Seidler, Phys. Rev. Lett. 87, 055702 (2001).

${ }^{22}$ E. Tyrode, C. M. Johnson, A. Kumpulainen, M. W. Rutland, and P. M. Claesson, J. Am. Chem. Soc. 127, 16848 (2005).

${ }^{23}$ Y. Qiu, N. Odendahl, A. Hudait, R. H. Mason, A. K. Bertram, F. Paesani, P. J. DeMott, and V. Molinero, J. Am. Chem. Soc. 139, 3052 (2017).

${ }^{24}$ D. A. Knopf and S. M. Forrester, J. Phys. Chem. A 115, 5579 (2011).

${ }^{25}$ D. R. Nutt and A. J. Stone, J. Chem. Phys. 119, 5670 (2003).

${ }^{26}$ E. Ochshorn and W. Cantrell, J. Chem. Phys. 124, 054714 (2006).

${ }^{27}$ B. Zobrist, T. Koop, B. P. Luo, C. Marcolli, and T. Peter, J. Phys. Chem. C 111, 2149 (2007).

${ }^{28}$ K. Wolfrum, H. Graener, and A. Laubereau, Chem. Phys. Lett. 213, 41 (1993).

${ }^{29}$ K. Wolfrum and A. Laubereau, Chem. Phys. Lett. 228, 83 (1994).

${ }^{30}$ C. D. Stanners, Q. Du, R. P. Chin, P. Cremer, G. A. Somorjai, and Y. R. Shen, Chem. Phys. Lett. 232, 407 (1995).

${ }^{31}$ R. Braun, B. D. Casson, and C. D. Bain, Chem. Phys. Lett. 245, 326 (1995).

${ }^{32}$ B. D. Casson, R. Braun, and C. D. Bain, Faraday Discuss. 104, 209 (1996).

${ }^{33}$ G. R. Bell, C. D. Bain, and R. N. Ward, J. Chem. Soc., Faraday Trans. 92, 515 (1996).

${ }^{34}$ Y.-C. Wen, S. Zha, X. Liu, S. Yang, P. Guo, G. Shi, H. Fang, Y. R. Shen, and C. Tian, Phys. Rev. Lett. 116, 016101 (2016).

${ }^{35}$ Y.-C. Wen, S. Zha, C. Tian, and Y. R. Shen, J. Phys. Chem. C 120, 15224 (2016).

${ }^{36}$ S. K. Reddy, R. Thiraux, B. A. W. Rudd, L. Lin, T. Adel, T. Joutsuka, F. M. Geiger, H. C. Allen, A. Morita, and F. Paesani, Chem 4, 1629 (2018).

${ }^{37}$ S. Ong, X. Zhao, and K. B. Eisenthal, Chem. Phys. Lett. 191, 327 (1992).

${ }^{38}$ P. Fischer, A. D. Buckingham, K. Beckwitt, D. S. Wiersma, and F. W. Wise, Phys. Rev. Lett. 91, 173901 (2003). 
${ }^{39}$ C. Neipert and B. Space, J. Chem. Phys. 125, 224706 (2006).

${ }^{40}$ X. Chen, J. Wang, Z. Paszti, F. Wang, J. N. Schrauben, V. V. Tarabara, A. H. Schmaier, and Z. Chen, Anal. Bioanal. Chem. 388, 65 (2007).

${ }^{41}$ A. Eftekhari-Bafrooei and E. Borguet, J. Phys. Chem. Lett. 2, 1353 (2011).

${ }^{42}$ K. C. Jena, P. A. Covert, and D. K. Hore, J. Phys. Chem. Lett. 2, 1056 (2011).

${ }^{43}$ P. Koelsch, M. I. Muglali, M. Rohwerder, and A. Erbe, J. Opt. Soc. Am. B 30, 219 (2013).

${ }^{44}$ A. J. Green and B. Space, J. Phys. Chem. B 119, 9219 (2015).

${ }^{45}$ P. E. Ohno, S. A. Saslow, H.-f. Wang, and F. M. Geiger, Nat. Commun. 7, 13587 (2016).

${ }^{46}$ T. Ishiyama, D. Terada, and A. Morita, J. Phys. Chem. Lett. 7, 216 (2016).

${ }^{47}$ A. Myalitsin, S. Urashima, S. Nihonyanagi, S. Yamaguchi, and T. Tahara, J. Phys. Chem. C 120, 9357 (2016).

${ }^{48}$ C. Humbert, B. Busson, and A. Tadjeddine, J. Phys. Chem. C 120, 16211 (2016).

${ }^{49}$ N. Ghosh, A. K. Singh, and J. A. Mondal, J. Phys. Chem. C 120, 23596 (2016).

${ }^{50}$ H.-F. Wang, Prog. Surf. Sci. 91, 155 (2016).

${ }^{51}$ P. E. Ohno, H.-f. Wang, and F. M. Geiger, Nat. Commun. 8, 1032 (2017).

${ }^{52}$ P. E. Ohno, H.-f. Wang, F. Paesani, J. L. Skinner, and F. M. Geiger, J. Phys. Chem. A 122, 4457 (2018).

${ }^{53}$ T. Joutsuka, T. Hirana, M. Sprik, and A. Morita, Phys. Chem. Chem. Phys. 20, 3040 (2018).

${ }^{54}$ D. R. Moberg, S. C. Straight, and F. Paesani, J. Phys. Chem. B 122, 4356 (2018).

${ }^{55}$ L. Martnez, R. Andrade, E. G. Birgin, and J. M. Martnez, J. Comput. Chem. 30, 2157 (2009).

${ }^{56}$ W. L. Jorgensen, D. S. Maxwell, and J. Tirado-Rives, J. Am. Chem. Soc. 118, 11225 (1996).

${ }^{57}$ G. A. Kaminski, R. A. Friesner, J. Tirado-Rives, and W. L. Jorgensen, J. Phys. Chem. B 105, 6474 (2001).

${ }^{58}$ J. L. Abascal and C. Vega, J. Chem. Phys. 123, 234505 (2005).

${ }^{59}$ S. Plimpton, J. Comput. Phys. 117, 1 (1995).

${ }^{60}$ S. Nosé, J. Chem. Phys. 81, 511 (1984).

${ }^{61}$ G. J. Martyna, M. L. Klein, and M. Tuckerman, J. Chem. Phys. 97, 2635 (1992).

${ }^{62}$ S. Habershon, T. E. Markland, and D. E. Manolopoulos, J. Chem. Phys. 131, 024501 (2009). 
${ }^{63}$ G. R. Medders and F. Paesani, J. Chem. Phys. 142, 212411 (2015).

${ }^{64}$ A. Morita and J. T. Hynes, J. Phys. Chem. B 106, 673 (2002).

${ }^{65}$ R. Kusaka, T. Ishiyama, S. Nihonyanagi, A. Morita, and T. Tahara, Phys. Chem. Chem. Phys. 20, 3002 (2018).

${ }^{66}$ G. R. Medders and F. Paesani, J. Chem. Theory Comput. 9, 4844 (2013).

${ }^{67}$ G. R. Medders and F. Paesani, J. Chem. Theory Comput. 11, 1145 (2015).

${ }^{68}$ V. Babin, C. Leforestier, and F. Paesani, J. Chem. Theory Comput. 9, 5395 (2013).

${ }^{69}$ G. R. Medders, V. Babin, and F. Paesani, J. Chem. Theory Comput. 9, 1103 (2013).

${ }^{70}$ V. Babin, G. R. Medders, and F. Paesani, J. Chem. Theory Comput. 10, 1599 (2014).

${ }^{71}$ S. E. Brown, A. W. Gtz, X. Cheng, R. P. Steele, V. A. Mandelshtam, and F. Paesani, J. Am. Chem. Soc. 139, 7082 (2017).

${ }^{72}$ G. R. Medders and F. Paesani, J. Am. Chem. Soc. 138, 3912 (2016).

${ }^{73}$ D. R. Moberg, S. C. Straight, C. Knight, and F. Paesani, J. Phys. Chem. Lett. 8, 2579 (2017).

${ }^{74}$ Y. Nagata and S. Mukamel, J. Am. Chem. Soc. 132, 6434 (2010).

${ }^{75}$ R. S. Taylor, L. X. Dang, and B. C. Garrett, J. Phys. Chem. 100, 11720 (1996).

${ }^{76}$ A. Morita and J. T. Hynes, Chem. Phys. 258, 371 (2000).

${ }^{77}$ I. V. Stiopkin, C. Weeraman, P. A. Pieniazek, F. Y. Shalhout, J. L. Skinner, and A. V. Benderskii, Nature 474, 192 (2011).

${ }^{78}$ M. Bonn, Y. Nagata, and E. H. G. Backus, Angew. Chem. 54, 5560 (2015).

${ }^{79}$ S. Yamaguchi, J. Chem. Phys. 143, 034202 (2015).

${ }^{80}$ S. Nihonyanagi, R. Kusaka, K.-i. Inoue, A. Adhikari, S. Yamaguchi, and T. Tahara, J. Chem. Phys. 143, 124707 (2015).

${ }^{81}$ E. E. Fileti, P. Chaudhuri, and S. Canuto, Chem. Phys. Lett. 400, 494 (2004).

${ }^{82}$ J. Towns, T. Cockerill, M. Dahan, I. Foster, K. Gaither, A. Grimshaw, V. Hazlewood, S. Lathrop, D. Lifka, G. D. Peterson, et al., Comput. Sci. Eng. 16, 62 (2014). 